\title{
Design of Automobile Body Welding Fixture
}

\author{
Liu $\mathrm{Xu}^{1,}$, , Chao $\mathrm{Ma}^{1}$ and Xia Xie ${ }^{1}$ \\ ${ }^{1}$ Military Transportation University, Tianjin 300161, China \\ axuliu333@sina.com
}

\begin{abstract}
Keywords: Automobile Body;Welding;Fixture
Abstract. In automobile industry, automobile welding fixture is component important to auxiliary welding. The automobile body is welded by multiple complex sheet stamping parts and other auxiliary workpiece. In the process, supporting role of welding fixture is an important step. In this paper, the fixture welding was designed and analyzed briefly.
\end{abstract}

\section{Introduction}

The cars are common means of transport in their daily lives. In the production of automotive factories, design, manufacturing, detection, sale, and after-sales service account for most of the automotive industry. In this part of the automobile manufacturing, auxiliary car body welding fixture is a very important role to ensure that the body mass. After the end of the car body design, designer in accordance with the mechanical properties of materials and safety performance, the design of the fixture.

Designer received the orders of the fixture design. According to the digital model of the car and welding point, and then split process. Consists of the following sections: The sum of the front wall assembly, The sum of the after wall assembly, The left side of the sum of the body assembly, The right side of the sum of the body assembly, and The sum of the chassis assembly. Below for each part of the respective split, split into two or three parts of a group of small modules. Split of the module must ensure that does not interfere with other parts.

Design department according to the process arrangement period and designers to design in accordance with the order. The fixture of each unit must achieve the required accuracy, otherwise it will affect the accuracy of the overall body.

According to split good plank separate design, in the design process, each fixture has floor, fixture, electrical system composition, floor is all fixture installation and positioning itself place. In order to facilitate the design and testing, but also in the floor that reference hole and auxiliary reference hole. Floor below are easy to moving universal wheel. Car fixture design is the design department according to the manufacturer to provide the data model design work.The flow chat shows in Fig.1.

\section{Software of Fixture Design}

In the design process will be applied to a series of three dimensional design software, and now most of the machinery industry, application software have UG, Pro/e, MDT, AutoCAD, etc. But in fixture design software design industry is the largest of the application of MDT this software.

MDT this software full name is the Mechanical Desktop, which also contains the AutoCAD software. MDT software interface with AutoCAD similar, some functions also have the same part, but MDT software loading a lot of special module, for example three dimensional pattern, calculation function and so on relatively new function, for the enterprise to carry on the design of the subsequent problems such as container handling for weight computation for solving the problems of the all have very good help. Inside this to a special explanation is MDT software parts modelling, the function of the components of fixture design is very convenient, whether parts combination, merger or parts, between the parts assembly practicability and find sex is very strong, won't appear parts confusion or it is not convenient to find the problem. 


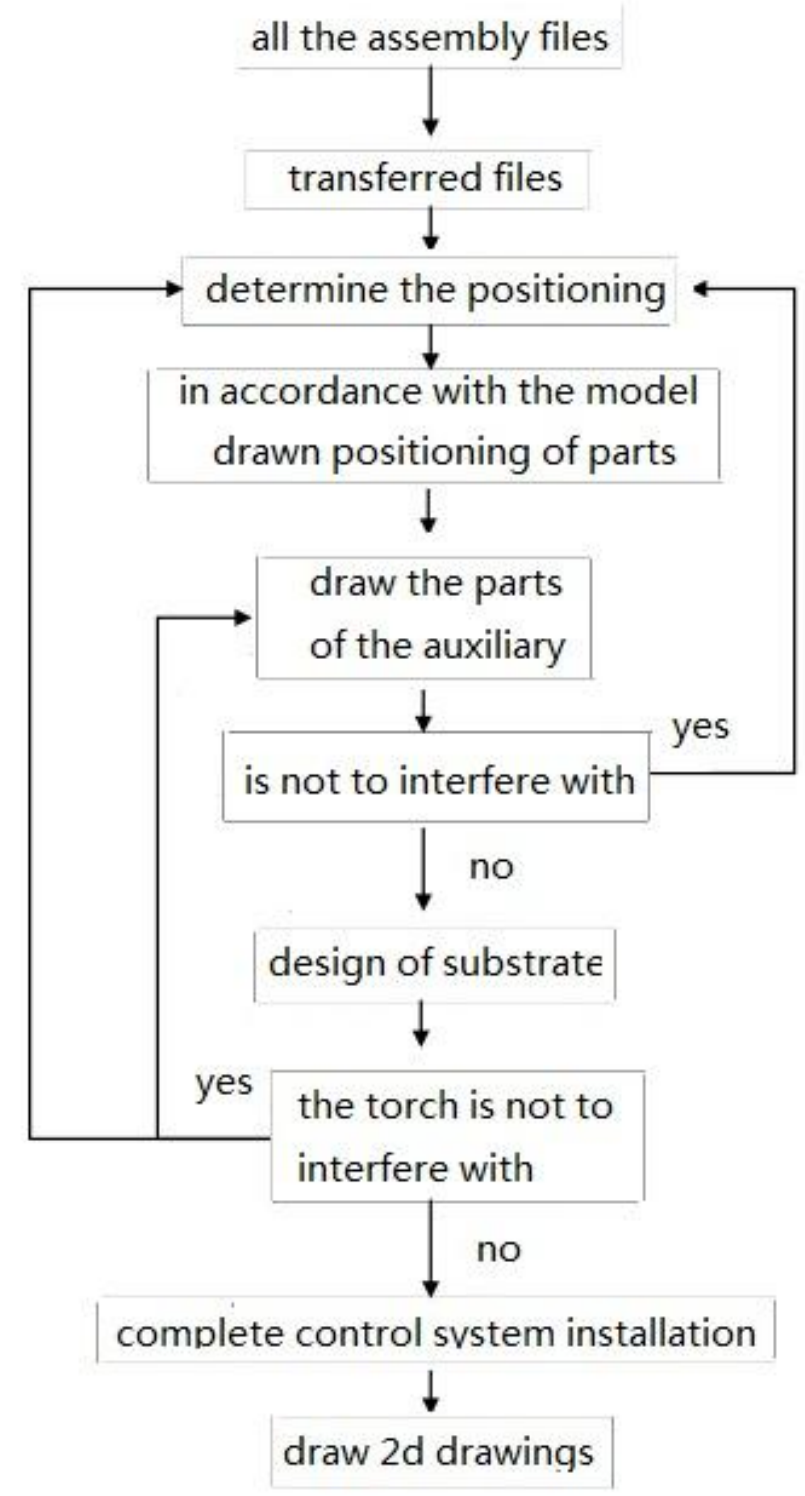

The design process

Fig.1 The Design Process

\section{Process of Automobile Fixture Design}

In developing a design, according to the resolution process has good body da weldments found, using UG, Pro/e, such as design software in the da. Igs formats derived, and then into the MDT. In the inside this common problem is:

The fixture design to avoid solder joint position, the position of the fixture is positioned to get out of the way solder joints, without spot place fixed installation. There in the design should pay attention to in the key of solder joints near needs to locate the position of key points to put a fixture, this is often the group among key points of fixture design, so that can reduce welding deformation,

In the positioning of body plate, based on the car body positioning hole as positioning reference. The designer should make full use of the body on the board hole as their own fixture design of hole 
reference to locate and support, guarantee the body during the welding process of the solder joint accurately.

The design of fixture and body contact support orientation block, a clamping piece, its size shape basically is fixed, is a L or is the TuZiXing. Only in specific contact body plate place according to the specific shape body plate by body cutting line in MDT software on SanShiTu projection, to draw support block and clamping block diagram. The map, in nc machine tool processing, work out to meet the requirements of positioning support clamping block. This process out of the surface of the parts size and shape and body contact surface shape is consistent, supporting clamping block shape directly affect the outside of the body processing quality, it is very key design steps.

The design of fixture requirements in fixture open state body board to be able smoothly from the fixture to take, this needs in the process of fixture design choose appropriate open stroke to determine the cylinder, make open after the clamping piece and the car body plate edge have $20 \sim 30 \mathrm{~mm}$ space. Preliminary design is completed, the assembly design test, see each aspect is appropriate, such as cylinder whether it can be opened, can take out on the workpiece, assignments, height is in compliance with the requirements of the human body engineering satisfies human body work habits, whether the welding torch can be convenient concise of solder joint operation. If no problem, which can make a control cylinder work of electric gas circuit design.

\section{Field Installation}

Workers in accordance with the drawings, installation, commissioning and testing. Factory Coordinate testing to test the installation position is consistent with the original size.If the position is inconsistent, but also manual debugging. The mounting fixture is detected whether the impact of the welding of the body.

\section{Welding Problems and Test}

For each fixture for test weld, that is not a problem to check work flow. Including the time rhythm is in accordance with the design requirement, welding out the position of the solder joints are accurate, workpiece have large welding thermal deformation.

If problems for feedback, to design.

\section{Thinking of the fixture}

As far as possible in the design process, selection criteria, such as the L-shaped block can be highly standardized design. Positioning support, clamping block, cylinder support arm can be made to standard parts, and then processed.Designers and workers to communicate in a timely manner. Will appear in a variety of design and process problems, if there is a problem we have to communicate in a timely manner in order to avoid duplication of production and waste of materials and working hours

Although the level of our machining development, but compared to developed countries, there are still gaps.

\section{Conclusion}

The plant should strive to introduce and improve the technology, learning new knowledge, the manufacturing level to catch up with developed countries as soon as possible.

\section{References}

[1] Li Zhao, Xubo Liu, Hongzhang Liu: Welding, (2006)No.8, p52-53.

[2] Lifeng Xu: Occupational Circle, (2007)No.1, p134-136.

[3] Hai You: Mechanical Engineer, Vol.23 (2007)No.7, p68-69. 